03

\title{
Простая теория многокомпонентной диффузии и моделирование горения стехиометрической смеси $\mathrm{H}_{2} / \mathrm{O}_{2}$
}

\author{
( К.О. Сабденов, М. Ерзада, А.Т. Сулейменов \\ Евразийский национальный университет им. Л.Н. Гумилева, \\ 010008 Нур-Султан, Казахстан \\ e-mail: sabdenovko@yandex.kz
}

Поступило в Редакцию 29 июня 2021 г.

В окончательной редакции 6 октября 2021 г.

Принято к публикации 7 октября 2021 г.

Предложена теория многокомпонентной диффузии на основе закона Фика, где при записи уравнений Максвелла-Стефана многокомпонентная смесь представляется состоящей из двух компонент: выделенного вещества и всех остальных со средними характеристиками. Число коэффициентов диффузии существенно снижается, указан способ их вычисления, и они сильно зависят от концентрации компонентов смеси. На основе результатов этой теории проведено моделирование горения стехиометрической смеси $\mathrm{H}_{2} / \mathrm{O}_{2}$. Проведен анализ влияния изменения состава смеси на процессы переноса, температуру и скорость горения.

Ключевые слова: газовые смеси, многокомпонентная диффузия, молярная масса, химическая работа, скорость горения.

DOI: 10.21883/JTF.2022.01.51846.198-21

\section{Введение}

При протекании химических реакций в газе происходит изменение его состава, как следствие меняется и средняя молярная масса газа. Здесь можно говорить о термодинамике химически реагирующей среды, свойства которой далеки от свойств среды, представляющей собой химически инертную смесь различных веществ. При горении высокоэнергетических материалов изменение молярной массы может быть существенной и оказывать сильное влияние на процессы горения $[1,2]$. Анализ этого вопроса показал важную роль химической работы, в законе сохранения энергии она появляется вследствие исчезновения одних сортов молекул и появления других $[3,4]$, и с энергетической точки зрения химическая работа связана с изменением теплоемкости в ходе химической реакции $[5,6]$. Но изменение состава газа отражается и на процессах переноса компонентов газовой смеси. В работе [6] этот вопрос не обсуждался, и исследование проводилось фактически в предположении независимости коэффициентов диффузии от состава смеси, а также в грубом приближении они считались равными для молекул $\mathrm{H}_{2}$ и $\mathrm{O}_{2}$. Поэтому представляет интерес влияние совместного действия химической работы и изменения коэффициентов диффузии на медленное горение стехиометрической смеси $\mathrm{H}_{2} / \mathrm{O}_{2}$. Этот вопрос необходимо сначала рассмотреть на простой модели реакции с химическим уравнением $\mathrm{H}_{2}+0.5 \mathrm{O}_{2} \rightarrow \mathrm{H}_{2} \mathrm{O}$. В сложных моделях [7-9], где детально рассматриваются химические реакции, процессы столкновений и перенос атомов, молекул и других промежуточных продуктов реакций, химическая работа не учитывается и эта физическая величина нигде не упоминается. Поэтому модели $[7,8]$, использующие программные коды CHEMKIN и др., можно пока оставить на перспективу. То же самое относится к сложным моделям горения, включающим частично методы молекулярной динамики, статистической физики и частично методы механики сплошной среды [9], нейронные сети $[10,11]$. Решение множества уравнений таких моделей требует очень больших вычислительных мощностей и объемов работы. В зависимости от попыток добиться точности моделирования приходится иметь дело с процессами, характеризующимися масштабами времени от $10^{-12}$ до нескольких секунд. В особенности это относится к образованию химических связей, поскольку здесь необходимо принимать во внимание не только энергии сталкивающихся частиц, но и их взаимную ориентацию в пространстве, а также свойства третьей частицы, уносящей избыток энергии. В работе [11] этот вопрос решается только частично, поэтому принятые в них константы химических реакций имеют лишь приближенный характер.

В отличие от таких сложных моделей, простые модели привлекательны способностью правильно описывать многие важные свойства горения. Они содержат минимум относительно легко определяемых входных параметров, с ними несложно проводить параметрический анализ. На основе полученных с помощью моделей теоретических данных можно делать полезные для практики выводы. Простота модели зависит не только от выбранного химического уравнения, но еще и от способа описания диффузионного переноса в смеси трех и более компонент. 


\section{1. Простая теория многокомпонентной диффузии}

Строгая математическая теория многокомпонентной диффузии и методы расчета транспортных характеристик диффузионного переноса хорошо развиты [12-20], простое изложение этой теории дано в $[21,22]$. В смеси из $N$ компонент и при постоянном давлении $p$ в газе связь между мольными потоками $\mathbf{j}_{r, i}\left[\mathrm{~mol} \cdot \mathrm{m}^{-2} \cdot \mathrm{s}^{-1}\right]$ и относительными объемными концентрациями $r_{i}=p_{i} / p$, где $p_{i}$ - парциальное давление, дается системой уравнений Максвелла-Стефана [19-22]:

$$
\begin{gathered}
\sum_{k \neq i}^{N} \frac{1}{D_{i k}}\left(r_{i} \mathbf{j}_{r, k}-r_{k} \mathbf{j}_{r, i}\right)=\frac{p}{R T} \nabla r_{i}, \\
\sum_{k}^{N} \mu_{k} \mathbf{j}_{r, k}=0 ; \quad i=1,2, \ldots, N .
\end{gathered}
$$

Поскольку при $p=$ const, система (1) относительно неизвестных потоков линейно зависима, и одно уравнение из (1) выбрасывается. Бинарные коэффициенты диффузии $D_{i k}$ определены равенствами

$$
\begin{gathered}
D_{i k}=D_{k i}=\frac{R^{2} T^{2}}{p N_{A} \mu_{i k}\langle\sigma v\rangle_{i k}}, \\
\mu_{i k}=\frac{\mu_{i} \mu_{k}}{\mu_{i}+\mu_{k}}, i, k=1,2,3 \ldots,
\end{gathered}
$$

где $R$ - универсальная газовая постоянная, $T-$ температура, $N_{A}$ - число Авогадро, $\sigma$ - сечение рассеяния, $v$ - средняя скорость относительного движения сталкивающихся частиц (молекул, атомов и др.), $\mu_{i}$ - молярная масса $i$-й компоненты смеси. Процессы переноса в смеси из $N$ компонент характеризуется

$$
\frac{N(N-1)}{2}
$$

различными бинарными коэффициентами диффузии. В теории основное внимание уделяется комплексу $\langle\sigma v\rangle_{i k}$, он определяется решением задачи о рассеянии двух частиц разных веществ при их однократном столкновении [12-15,21-23], а также оценке величины элементов обратной матрицы системы (1) и (2) для выяснения роли перекрестных эффектов с целью упрощения этих уравнений [16-18].

Если $\nabla p \neq 0$, то условие сохранения потока импульса (2) становится лишним, и диффузионные потоки в явном виде зависят от $\nabla p$. В уравнениях (1) не учитывается термодиффузия, бародиффузия и другие явления $[16,17,21]$, в рассматриваемых ниже задачах медленного горения они являются слабыми эффектами. Рассматривая (1) и (2) как алгебраические уравнения относительно неизвестных потоков $\mathbf{j}_{r, i}$, последние можно выразить через градиенты концентрации [22,23]. В частности, для бинарной диффузии при смешении двух веществ 1 и 2 получаются уравнения

$$
\begin{gathered}
\mathbf{j}_{r, 1}=-\frac{\mu_{2}}{\mu_{\text {mix }}} \frac{p D_{12}}{R T} \nabla r_{1}=-\frac{\mu_{2}}{\mu_{\text {mix }}^{2}} D_{12} \rho \nabla r_{1}, \\
\mathbf{j}_{r, 2}=-\frac{\mu_{1}}{\mu_{\text {mix }}} \frac{p D_{21}}{R T} \nabla r_{2}=-\frac{\mu_{1}}{\mu_{\text {mix }}^{2}} D_{21} \rho \nabla r_{2}, \\
\mu_{\text {mix }}=r_{1} \mu_{1}+r_{2} \mu_{2},
\end{gathered}
$$

где $\rho$ - плотность смеси газов, здесь и ниже $\mu_{\operatorname{mix}}-$ средняя молярная масса всей смеси. Переход к массовым концентрациям $g_{1}, g_{2}$ и к массовым потокам $\mathbf{j}_{g, 1}, \mathbf{j}_{g, 2}$ выполняется использованием уравнений связи [24]:

$$
r_{1}=\frac{\mu_{\mathrm{mix}}}{\mu_{1}} g_{1}, \quad r_{2}=\frac{\mu_{\mathrm{mix}}}{\mu_{2}} g_{2}
$$

а также равенств $\mathbf{j}_{g, 1}=\mu_{1} \mathbf{j}_{r, 1}, \mathbf{j}_{g, 2}=\mu_{1} \mathbf{j}_{r, 2}$. После вычисление градиентов

$$
\nabla r_{1}=\frac{\mu_{\text {mix }}^{2}}{\mu_{1} \mu_{2}} \nabla g_{1}, \quad \nabla r_{2}=\frac{\mu_{\text {mix }}^{2}}{\mu_{1} \mu_{2}} \nabla g_{2}
$$

и выполнения несложных преобразований получаются законы Фика ${ }^{1}[22,23]$ :

$$
\mathbf{j}_{g, 1}=-\rho D_{12} \nabla g_{1}, \mathbf{j}_{g, 2}=-\rho D_{21} \nabla g_{2},
$$

В случае числа компонент $N>2$ диффузионный поток каждого из веществ зависит от градиентов концентрации всех остальных компонентов и определяется еще и самими концентрациями. В результате получаются сложные и громоздкие выражения для диффузионных потоков

$$
\mathbf{j}_{r, 1}=\sum_{k} M_{i k} \nabla r_{k}, i=1,2, \ldots
$$

где $M_{i k}$ - элементы обратной матрицы уравнений (1) и (2). Квадратичный рост числа бинарных коэффициентов диффузии от $N$ и сильно усложняющиеся матричные элементы $M_{i k}$ делают сомнительным перспективность подхода на основе уравнений (1) и (2). Возможно, по этой причине приведенные выше результаты не находят применения в физике горения [26-29]. В этих и многих других работах применяется простой закон Фика, где поток вещества с номером $i$ зависит только от градиента его концентрации. Ниже показано, что такой способ описания диффузионного переноса имеет теоретическое обоснование.

Предлагаемая модель диффузии опирается на свойства бинарной смеси, или на двухкомпонентном представлении смеси газа: выбранного вещества с номером $i$ и всех оставшихся со средней молярной массой $\mu_{\text {oth }}$, парциальные давления компонентов $p_{i}$ и $p_{\text {oth }}$ (others -

\footnotetext{
${ }^{1}$ Бинарные коэффициенты диффузии при наличии мелкомасштабных конвективных вихрей в среде могут зависеть от векторного потенциала циркуляции, в результате вместо коэффициентов $D_{12}=D_{21}$ следует использовать эффективное значение $D_{\text {eff }}[25]$.
} 
остальные). Уравнения Максвелла-Стефана при постоянном давлении $p=p_{i}+p_{\text {oth }}=$ const записываются как для бинарной смеси, но каждому номеру $i$ соответствуют различные $\mu_{\text {oth }}$ и $p_{\text {oth }}$ :

$$
\begin{gathered}
\frac{1}{D_{i, \text { oth }}}\left(r_{i} \mathbf{j}_{r, \text { oth }}-r_{\text {oth }} \mathbf{j}_{r, i}\right)=\frac{p}{R T} \nabla r_{i}, \\
\mu_{i} \mathbf{j}_{r, \text { oth }}+\mu_{\text {oth }} \mathbf{j}_{r, i}=0 ; i=1,2, \ldots, N .
\end{gathered}
$$

Отсюда потоки легко находятся, и вместо (3) и (4) используются уравнения

$$
\begin{gathered}
\mathbf{j}_{r, i}=-\frac{\mu_{\mathrm{oth}}}{\mu_{\mathrm{mix}}^{2}} D_{i, \mathrm{oth}} \rho \nabla r_{i}, \mathbf{j}_{r, \mathrm{oth}}=-\frac{\mu_{i}}{\mu_{\mathrm{mix}}^{2}} D_{\mathrm{oth}, i} \rho \nabla r_{\mathrm{oth}}, \\
\frac{1}{\mu_{\mathrm{mix}}}=\sum_{k} \frac{g_{k}}{\mu_{k}} ; D_{i, \mathrm{oth}}=\frac{R^{2} T^{2}}{p N_{A} \mu_{i, \mathrm{oth}}\langle\sigma v\rangle_{i, \mathrm{oth}}}, \\
\mu_{i, \mathrm{oth}}=\frac{\mu_{i} \mu_{\mathrm{oth}}}{\mu_{i}+\mu_{\mathrm{oth}}}, i=1,2, \ldots, N .
\end{gathered}
$$

Коэффициенты диффузии обладают свойством симметрии $D_{i, \text { oth }}=D_{\text {oth }, i}$, для них удобно ввести обозначение $D_{i}$. Теперь для моделирования переноса $i$-го вещества в окружающей ее многокомпонентной среде используется закон Фика с одним коэффициентом диффузии $D_{i}$. Он имеет смысл бинарного коэффициента диффузии и характеризует перенос $i$-го компонента в этой среде. Поток $\mathbf{j}_{r}$, th интереса не представляет, он здесь играет вспомогательную роль.

Переход к концентрациям $g_{i}$ производится на основе уравнения связи [24]

$$
r_{i}=\frac{\mu_{\mathrm{mix}}}{\mu_{i}} g_{i}
$$

где $\mu_{\text {mix }}$ можно представить в виде

$$
\frac{1}{\mu_{\text {mix }}}=\frac{1}{\mu_{\mathrm{oth}}}+\left(\frac{1}{\mu_{i}}-\frac{1}{\mu_{\mathrm{oth}}}\right) g_{i} .
$$

Тогда вычисление градиента дает выражение

$$
\nabla r_{i}=\frac{\mu_{\mathrm{mix}}}{\mu_{i}} \nabla g_{i}+\frac{g_{i}}{\mu_{i}} \nabla \mu_{\mathrm{mix}}
$$

В градиенте от молярной массы смеси содержатся два члена, пропорциональные $\nabla \mu_{\text {oth }}$ и $\nabla g_{i}$ :

$$
\nabla \mu_{\text {mix }}=-\mu_{\text {mix }}^{2}\left[-\frac{1-g_{i}}{\mu_{\text {oth }}^{2}} \nabla \mu_{\mathrm{oth}}+\left(\frac{1}{\mu_{i}}-\frac{1}{\mu_{\mathrm{oth}}}\right) \nabla g_{i}\right] .
$$

Ниже предполагается слабое изменение относительной молярной массы по сравнению с концентрацией, или выполнение неравенства

$$
\frac{1}{\mu_{\text {oth }}} \nabla \mu_{\text {oth }} \ll \nabla g_{i}
$$

Это позволяет записать приближенное равенство

$$
\nabla \mu_{\mathrm{mix}} \approx-\mu_{\mathrm{mix}}^{2}\left(\frac{1}{\mu_{i}}-\frac{1}{\mu_{\mathrm{oth}}}\right) \nabla g_{i}
$$

после этого можно получить связь между градиентами объемной и массовой концентраций [22]:

$$
\nabla r_{i}=\frac{\mu_{\text {mix }}^{2}}{\mu_{i} \mu_{\mathrm{oth}}} \nabla g_{i}
$$

Тогда молярный поток запишется в виде

$$
\mathbf{j}_{r, i}=-\frac{1}{\mu_{i}} D_{i} \rho \nabla g_{i} .
$$

Векторы компонентов массового потока $\mathbf{j}_{g, i}=\mu_{i} \mathbf{j}_{r, i}$ связаны с относительными массовыми концентрациями $g_{i}$ :

$$
\mathbf{j}_{g, i}=-\rho D_{i} \nabla g_{i}, \quad i=1,2,3, \ldots .
$$

Ниже предлагается приближенный способ определения $D_{i}$, например, для вещества с номером 1 можно записать

$$
D_{1}=\frac{R^{2} T^{2}}{p N_{A} \mu_{1}\langle\sigma \nu\rangle_{1, \mathrm{oth}}}\left(1+\frac{\mu_{1}}{\mu_{\mathrm{oth}}}\right), \frac{1}{\mu_{\mathrm{oth}}}=\frac{1}{1-g_{1}} \sum_{k \neq 1} \frac{g_{k}}{\mu_{k}} .
$$

Множитель $\left(1-g_{1}\right)^{-1}$ позволяет пересчитывать концентрации при переходе от систем из $N-1$ частиц к системе из $N$ частиц. Параметр $\langle\sigma v\rangle_{1 \text {,oth }}$ характеризует одно столкновение частицы вещества с номером 1 со всеми остальными частицами. Его точный расчет сложнее, чем для двух частиц с заданным потенциалом взаимодействия, и здесь он определяется как среднее по объему от всех возможных однократных столкновений:

$$
\begin{aligned}
\langle\sigma v\rangle_{1, \mathrm{oth}} & =\frac{\langle\sigma v\rangle_{1,2} r_{2}+\langle\sigma v\rangle_{1,3} r_{3}+\ldots+\langle\sigma v\rangle_{1, N} r_{N}}{r_{2}+r_{3}+\ldots+r_{N}} \\
& =\frac{\sum_{k \neq 1}\langle\sigma v\rangle_{1, k} r_{k}}{\sum_{k \neq 1} r_{k}} .
\end{aligned}
$$

Здесь параметры столкновений $\langle\sigma v\rangle_{1, k}$ определяются так же, как и в теории Максвелла-Стефана. Относительные объемные концентрации $r_{i}, i=2,3, \ldots$ с точностью до константы являются вероятностями однократного столкновения частицы вещества с омером 1 с частицами веществ с номерами 2,3,... Сумма в знаменателе дроби добавлена для нормировки вероятностей, она равна $1-r_{1}$, поэтому в упрощенном виде получается

$$
\langle\sigma v\rangle_{1, \text { oth }}=\frac{1}{1-r_{1}} \sum_{k \neq 1}\langle\sigma v\rangle_{1, k} r_{k}
$$

Аналогичные рассуждения проводятся для всех оставшихся коэффициентов диффузии, и, в конечном счете, для произвольного номера $i$ коэффициент диффузии определяется равенствами

$$
\begin{gathered}
D_{i}=\frac{R^{2} T^{2}}{p N_{A} \mu_{i}\langle\sigma v\rangle_{1, \text { oth }}}\left(1+\frac{\mu_{i}}{\mu_{\text {oth }}}\right), \frac{1}{\mu_{\text {oth }}}=\frac{1}{1-g_{i}} \sum_{k \neq i} \frac{g_{k}}{\mu_{k}}, \\
\langle\sigma v\rangle_{i, \text { oth }}=\frac{1}{1-r_{i}} \sum_{k \neq i}\langle\sigma v\rangle_{i k} r_{k}, \\
r_{i}=\frac{\mu_{\text {mix }}}{\mu_{i}} g_{i}, \frac{1}{\mu_{\text {mix }}}=\sum_{k} \frac{g_{k}}{\mu_{k}} .
\end{gathered}
$$


Уравнения (5) и (6) в частном случае бинарной диффузии совпадают с теми, что получаются из систем (1) и (2). Действительно, для смеси из двух веществ 1 и 2 можно записать

$$
\begin{gathered}
\frac{1}{\mu_{\text {mix }, 1}}=\frac{1}{1-g_{1}} \frac{g_{2}}{\mu_{2}}=\frac{1}{\mu_{2}}, \\
\langle\sigma v\rangle_{1, \text { oth }}=\frac{1}{1-r_{1}}\langle\sigma v\rangle_{12} r_{2}=\langle\sigma v\rangle_{12}, \\
D_{1}=\frac{R^{2} T^{2}}{p N_{A} \mu_{1}\langle\sigma v\rangle_{12}}\left(1+\frac{\mu_{1}}{\mu_{2}}\right)=\frac{R^{2} T^{2}}{p N_{A}\langle\sigma v\rangle_{12}} \frac{\mu_{1}+\mu_{2}}{\mu_{1} \mu_{2}} .
\end{gathered}
$$

В смесях, где происходит сильное изменение молярной массы в результате химической реакции, множитель

$$
1+\frac{\mu_{i}}{\mu_{\text {oth }}}
$$

тоже меняется сильно. Например, реакция разложения взрывчатого вещества CL-20 сопровождается изменением молярной массы примерно в 16 раз [5,29]. Проведем оценку пределов возможных изменений комплекса

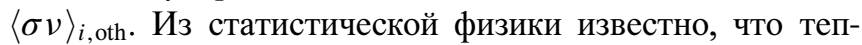
ловая скорость движения молекул обратно пропорциональна квадратному корню от их массы, или молярной массы $\mu$ :

$$
v \sim \frac{1}{\sqrt{\mu}} .
$$

Возможные квантовые эффекты не учитываются, молекулы представим в виде твердых шаров, тогда сечение $\sigma$ пропорционально квадрату диаметра молекул, и справедлива оценка с использованием объема молекулы $V$ :

$$
\sigma \sim V^{2 / 3} \sim \mu^{2 / 3}
$$

В результате находим, что

$$
\langle\sigma v\rangle_{i, \text { oth }} \sim\langle\sigma v\rangle_{i k} \sim \sigma v \sim \frac{\mu^{2 / 3}}{\sqrt{\mu}} \sim \mu^{1 / 6} .
$$

Это слабая зависимость и ее можно не принимать во внимание по сравнению с множителем в круглых скобках в (6). При изменении молярной массы смеси газов в 2 раза изменение $\langle\sigma v\rangle_{i, \text { oth }}$ составляет примерно $12 \%$. Таким образом, зависимостью $\langle\sigma v\rangle_{i, \text { oth }}$ от состава смеси можно пренебречь.

При моделировании горения коэффициенты диффузии (6) удобно определять относительно некоторого начального состояния с температурой $T_{0}$ и давлением $p_{0}$. Тогда уравнения (5) без учета слабой зависимости $\langle\sigma v\rangle_{i, \text { oth }}$ от молярной массы дополняются равенствами

$$
\begin{gathered}
D_{i}=D_{i, 0} \frac{p_{0}}{p}\left(\frac{T}{T_{0}}\right)^{2} G_{i}(g), \\
G_{i}(g)=\frac{1+\frac{\mu_{i}}{1-g_{i}} \sum_{k \neq i} \frac{g_{k}}{\mu_{k}}}{1+\frac{\mu_{i}}{1-g_{i}^{(0)}} \sum_{k \neq i} \frac{g_{k}^{(0)}}{\mu_{k}}}
\end{gathered}
$$

где $D_{i, 0}$ - const, $g_{i}(0)$ - значения концентраций в начальном состоянии. Для бинарной смеси функция $G_{i}(g)$ не зависит от изменения состава газовой смеси, для этого смесь должна состоять из трех и более компонент.

В итоге получено математически простое описание многокомпонентной диффузии. Кроме того, уменьшается число коэффициентов диффузии. Поскольку концентрации $g_{i}$ связаны равенством $g_{1}+g_{2}+\ldots+g_{N}=1$, достаточно знать $N-2$ коэффициентов диффузии (7) при $N>3$. Если $N=3$, то в химических реакциях, по крайней мере, две компоненты будут находиться в начальном или конечном состояниях в бинарном отношении с одним коэффициентом диффузии. Тогда число различных коэффициентов диффузии будет равно $N-2=1$.

\section{2. Моделирование горения смеси $\mathrm{H}_{2} / \mathrm{O}_{2}$}

Рассмотрим модель горения смеси веществ А и В с образованием продукта С на основе простого химического уравнения

$$
v_{\mathrm{A}} \mathrm{A}+v_{\mathrm{B}} \mathrm{B} \rightarrow v_{\mathrm{C}} \mathrm{C}
$$

где $v_{\mathrm{A}}, v_{\mathrm{B}}$ и $v_{\mathrm{C}}$ - стехиометрические коэффициенты. Существуют более сложные и точные модели реакции в смеси $\mathrm{H}_{2} / \mathrm{O}_{2}$, включающие от 19 [7] до $24[8,10,11]$ промежуточных химических превращений, они обладают высокой детальностью описания цепного характера химической реакции, но в них диффузионный перенос промежуточных продуктов рассматривается в сильно упрощенном виде. Поэтому ниже простая модель (8) используется в основном для анализа меры влияния коэффициентов диффузии на величину скорости горения.

Скорость химической реакции в законе Аррениуса содержит концентрации веществ А и В, поэтому достаточно рассматривать только их коэффициенты диффузии. Молярные массы компонентов А, В и С соответственно обозначены $\mu_{\mathrm{A}}, \mu_{\mathrm{B}}$ и $\mu_{\mathrm{C}}$. Для относительных массовых концентраций используются обозначения $g_{\mathrm{A}}, g_{\mathrm{B}}$ и $g_{\mathrm{C}}$. Давление постоянное и $p=p_{0}$. Поскольку $g_{\mathrm{C}}=1-g_{\mathrm{A}}-g_{\mathrm{B}}$, формулы (7) запишутся в виде

$$
\begin{gathered}
D_{\mathrm{A}}=D_{0}\left(\frac{T}{T_{0}}\right)^{2} G_{\mathrm{A}}, \\
G_{\mathrm{A}}=\frac{\mu_{\mathrm{B}}}{\mu_{\mathrm{A}}+\mu_{\mathrm{B}}}\left[1+\frac{\mu_{\mathrm{A}}}{\mu_{\mathrm{C}}}+\frac{\mu_{\mathrm{A}} g_{\mathrm{B}}}{1-g_{\mathrm{A}}}\left(\frac{1}{\mu_{\mathrm{B}}}-\frac{1}{\mu_{\mathrm{C}}}\right)\right], \\
D_{\mathrm{B}}=D_{0}\left(\frac{T}{T_{0}}\right)^{2} G_{\mathrm{B}}, \\
G_{\mathrm{B}}=\frac{\mu_{\mathrm{A}}}{\mu_{\mathrm{A}}+\mu_{\mathrm{B}}}\left[1+\frac{\mu_{\mathrm{B}}}{\mu_{\mathrm{C}}}+\frac{\mu_{\mathrm{B}} g_{\mathrm{A}}}{1-g_{\mathrm{B}}}\left(\frac{1}{\mu_{\mathrm{A}}}-\frac{1}{\mu_{\mathrm{C}}}\right)\right],
\end{gathered}
$$

$D_{0}-$ const.

Изменение состава смеси газов оказывает влияние и на среднюю теплопроводность $\lambda$. Существует большое 
число различных способов расчета $\lambda$ для смесей, но по сравнению с коэффициентом диффузии $\lambda$ слабо зависит от состава смеси. Поэтому здесь учитывается только зависимость от температуры в форме [29-32]

$$
\lambda=\lambda_{0} \frac{T}{T_{0}} .
$$

Составление уравнений для участвующих в реакции компонентов начинается с установления связи между изменениями парциальных плотностей $\rho_{\mathrm{A}}, \rho_{\mathrm{B}}, \rho_{\mathrm{C}}$ соответственно веществ $\mathrm{A}, \mathrm{B}$ и $\mathrm{C}$ и скоростью химической реакции $W$. В произвольном и малом объеме изменение каждого химически реагирующего компонента может произойти как за счет химического превращения, так и за счет потоков $\mathbf{j}_{g, \mathrm{~A}}, \mathbf{j}_{g, \mathrm{~B}}$ и $\mathbf{j}_{g, \mathrm{C}}$, другие возможные источники нехимической природы считаются отсутствующими. За малое время $d t$ происходят изменения плотностей $d \rho_{\mathrm{A}}, d \rho_{\mathrm{B}}, d \rho_{\mathrm{C}}$, но из них необходимо исключить унос вещества потоками из выделенного объема за время $d t$. Тогда оставшиеся части будут соответствовать действию химической реакции и должны выполняться условия пропорциональности

$$
\begin{aligned}
-\frac{d \rho_{\mathrm{A}}+\operatorname{divj}_{g, \mathrm{~A}} d t}{v_{\mathrm{A}} \mu_{\mathrm{A}}} & =-\frac{d \rho_{\mathrm{B}}+\operatorname{div}_{g, \mathrm{~B}} d t}{v_{\mathrm{B}} \mu_{\mathrm{B}}} \\
& =\frac{d \rho_{\mathrm{C}}+\operatorname{div}_{g, \mathrm{C}} d t}{v_{\mathrm{C}} \mu_{\mathrm{C}}}=W d t
\end{aligned}
$$

Для движущейся среды изменения плотностей включают конвективный перенос. Эти равенства можно рассматривать в качестве определения скорости химической реакции $W$. Они также служат для формулировки уравнений для парциальных плотностей.

Уравнение для температуры, согласно уравнению (8), включающее производство химической работы, получено в [6]. Его вывод основывается на следующих положениях:

- изменением давления при ламинарном горении можно пренебречь ( $p \approx$ const) [26,27];

- при изменении числа частиц $n_{i}$ молекул сорта $i$ $(i=1,2, \ldots)$ в смеси газов дифференциал внутренней энергии $U$ определяется как $[3,4]$

$$
d U=\sum_{i}\left(\frac{\partial U}{\partial n_{i}}\right)_{p, T} d n_{i}
$$

- внутренняя энергия смеси газов равна сумме внутренних энергий компонентов смеси,

$$
U=\sum_{i} c_{v, i} m_{i} T
$$

где $m_{i}, c_{v, i}$ - массы и теплоемкости при постоянном объеме веществ сорта $i, m_{i}=g_{i} m ; m$ - полная масса смеси. Дополнительно необходимо учесть зависимость газовой постоянной смеси $R_{g}$ от ее состава [24];
- теплоемкости $c_{v, i}$, а также теплоемкости при постоянном давлении $c_{p, i}$ можно приближенно считать постоянными.

Предполагается зависимость процессов только от одной пространственной координаты $x$. В общем случае для реакции (8) изменения внутренней энергии $U_{v}$, механической работы $W_{v}$ и тепла $Q_{v}$ в произвольном и малом объеме $v$ и за малое время $d t$ имеют вид

$$
\begin{gathered}
d U_{v}=v c_{v} \rho \frac{\partial T}{\partial t} d t+v \rho T \frac{\partial c_{v}}{\partial t} d t+v c_{v} T \frac{\partial \rho}{\partial t} d t \\
\frac{\partial c_{v}}{\partial t}=\left(c_{v, \mathrm{~A}}-c_{v, \mathrm{C}}\right) \frac{\partial g_{\mathrm{A}}}{\partial t}+\left(c_{v, \mathrm{~B}}-c_{v, \mathrm{C}}\right) \frac{\partial g_{\mathrm{B}}}{\partial t}, \\
d W_{v}=\frac{d p}{d t} v d t-R_{g} \rho \frac{d T}{d t} v d t \\
-\rho T\left(\left(R_{g, \mathrm{~A}}-R_{g, \mathrm{C}}\right) \frac{d g_{\mathrm{A}}}{d t}+\left(R_{g, \mathrm{~B}}-R_{g, \mathrm{C}}\right) \frac{d g_{\mathrm{B}}}{d t}\right) v d t \\
d Q_{v}=-\frac{\partial \rho u c_{v} T}{\partial x} v d t+\frac{\partial}{\partial x}\left(\lambda \frac{\partial T}{\partial x}\right) v d t+Q W_{c h} v d t
\end{gathered}
$$

Здесь $c_{v, \mathrm{~A}}, c_{v, \mathrm{~B}}$ и $c_{v, \mathrm{C}}$ - теплоемкости при постоянном объеме индивидуальных веществ А, В и $\mathrm{C} ; R_{g, \mathrm{~A}}$, $R_{g, \text { В }}$ и $R_{g, \text { С }}$ - их газовые постоянные; $Q$ и $W_{c h}-$ тепловой эффект и скорость химической реакции. Члены с производными от концентраций $g_{\mathrm{A}}$ и $g_{\mathrm{B}}$ определяют химическую работу, совершаемую при исчезновении молекул одного вещества и появлении молекул другого вещества.

Использование приведенных выражений для $d U_{v}, d W_{v}$ и $d Q_{v}$ в Первом законе термодинамики

$$
d U_{v}=d W_{v}+d Q_{v}
$$

после несложных преобразований дает уравнение для температуры. Способ получения остальных уравнений сохранения массы и отдельных веществ известен $[22,26,27]$. В результате, с учетом равенств (9) и принятых выше допущений, для одномерного по пространству процесса получаются следующие уравнения:

$$
\begin{gathered}
\frac{d T}{d t}=\frac{1}{c_{p} \rho} \frac{\partial}{\partial x}\left(\lambda \frac{\partial T}{\partial x}\right)+A_{\mathrm{A}}^{\prime}+A_{\mathrm{B}}^{\prime}+\frac{Q}{c_{p}} W^{\prime}, \\
A_{\mathrm{A}}^{\prime}=-T \frac{c_{p, \mathrm{~A}}-c_{p, \mathrm{C}}}{c_{p}} \frac{d g_{\mathrm{A}}}{d t}, A_{\mathrm{B}}^{\prime}=-T \frac{c_{p, \mathrm{~B}}-c_{p, \mathrm{C}}}{c_{p}} \frac{d g_{\mathrm{B}}}{d t} \\
\frac{d g_{\mathrm{A}}}{d t}=\frac{1}{\rho} \frac{\partial}{\partial x}\left(D_{\mathrm{A}} \rho \frac{\partial g_{\mathrm{A}}}{\partial x}\right)-v_{\mathrm{A}} \mu_{\mathrm{A}} W^{\prime} \\
\frac{d g_{\mathrm{B}}}{d t}=\frac{1}{\rho} \frac{\partial}{\partial x}\left(D_{\mathrm{B}} \rho \frac{\partial g_{\mathrm{B}}}{\partial x}\right)-v_{\mathrm{B}} \mu_{\mathrm{B}} W^{\prime}, \\
\frac{\partial \rho}{\partial t}+\frac{\partial \rho u}{\partial x}=0, W^{\prime}=\rho^{n-1} g_{\mathrm{A}}^{\nu_{\mathrm{A}}} g_{\mathrm{B}}^{\nu_{\mathrm{B}}} k_{0} \exp \left(-\frac{E}{R T}\right) \\
n=v_{\mathrm{A}}+v_{\mathrm{B}}, \\
c_{p}=\left(c_{p, \mathrm{~A}}-c_{p, \mathrm{C}}\right) g_{\mathrm{A}}+\left(c_{p, \mathrm{~B}}-c_{p, \mathrm{C}}\right) g_{\mathrm{B}}+c_{p, \mathrm{C}} .
\end{gathered}
$$


Фронт горения движется справа налево вдоль координаты $x$. Полная производная по времени $t$ определена как оператор

$$
\frac{d}{d t}=\frac{\partial}{\partial t}+u \frac{\partial}{\partial x}
$$

где $u$ - скорость газа. Кроме того, приняты обозначения: $\left.c_{p, \mathrm{~A}}, c_{p, \mathrm{~B}}\right), c_{p, \mathrm{C}}-$ теплоемкости при постоянном давлении веществ А, В и $\mathrm{C}$; $E$ - энергия активации.

Плотность $\rho$ находится из уравнения состояния газовой смеси:

$$
\rho=\frac{p_{0}}{R T} \mu_{\text {mix }}, \mu_{\text {mix }}=\left(\frac{g_{\mathrm{A}}}{\mu_{\mathrm{A}}}+\frac{g_{\mathrm{B}}}{\mu_{\mathrm{B}}}+\frac{1-g_{\mathrm{A}}-g_{\mathrm{B}}}{\mu_{\mathrm{C}}}\right)^{-1} .
$$

Компоненты смеси со стехиометрическим составом в начальном состоянии имеют концентрации

$$
g_{\mathrm{A}, 0}=\frac{v_{\mathrm{A}} \mu_{\mathrm{A}}}{v_{\mathrm{A}} \mu_{\mathrm{A}}+v_{\mathrm{B}} \mu_{\mathrm{B}}}, g_{\mathrm{B}, 0}=\frac{v_{\mathrm{B}} \mu_{\mathrm{B}}}{v_{\mathrm{A}} \mu_{\mathrm{A}}+v_{\mathrm{B}} \mu_{\mathrm{B}}} .
$$

Для уравнений (10) используются граничные условия

$$
\begin{gathered}
x=-\infty: T=T_{0}, g_{\mathrm{A}}=g_{\mathrm{A}, 0}, g_{\mathrm{B}}=g_{\mathrm{B}, 0}, u=u_{b} ; \\
x=+\infty: \frac{\partial T}{\partial x}=0, \frac{\partial g_{\mathrm{A}}}{\partial x}=0, \frac{\partial g_{\mathrm{B}}}{\partial x}=0 .
\end{gathered}
$$

Скорость горения $u_{b}$ определяется следующим обра-

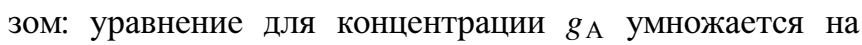
плотность и производится его интегрирование по всему пространству, в результате с использованием граничных условий получается выражение

$$
u_{b}=\frac{1}{g_{\mathrm{A}, 0} \rho_{0}} \int_{-\infty}^{+\infty} \rho\left(\frac{\partial g_{\mathrm{A}}}{\partial t}-v_{\mathrm{A}} \mu_{\mathrm{A}} W^{\prime}\right) d x
$$

где $\rho_{0}-$ начальная плотность.

В случае стехиометрической смеси водород/кислород $\mathrm{A}-\mathrm{H}_{2}, \mathrm{~B}-\mathrm{O}_{2}, \mathrm{C}-\mathrm{H}_{2} \mathrm{O} ; v_{\mathrm{A}}=2, v_{\mathrm{B}}=1 ; v_{\mathrm{C}}=2$; молярные массы $-\mu_{\mathrm{A}}=2, \mu_{\mathrm{B}}=32, \mu_{\mathrm{C}}=18 \mathrm{~g} / \mathrm{mol}$. Уравнение температуры в (10) получено в предположении постоянства индивидуальных теплоемкостей веществ $c_{p, \mathrm{~A}}$, $c_{p, \mathrm{~B}}$ и $c_{p, \mathrm{C}}\left[\mathrm{J} \cdot \mathrm{kg}^{-1} \cdot \mathrm{K}^{-1}\right]$, но они могут применяться и при их слабой зависимости от температуры. На основе экспериментальных данных [33,34] приняты следующие зависимости от температуры [6]:

$$
\begin{gathered}
c_{p, \mathrm{~A}}=14200+0.22(T-273)^{1.2}, \\
c_{p, \mathrm{~B}}=910+4.0(T-273)^{0.55}, \\
c_{p, \mathrm{C}}=1866+1.8(T-273)^{0.8} .
\end{gathered}
$$

Остальные входные параметры:

$T_{0}=293 \mathrm{~K}, \quad p_{0}=1.01 \cdot 10^{5} \mathrm{~Pa} ; \quad Q=285 \mathrm{~kJ} \cdot \mathrm{mol}^{-1}$; $E=175 \mathrm{~kJ} \cdot \mathrm{mol}^{-1} \cdot \mathrm{K}^{-1} ; k_{0}=8.64 \cdot 10^{16} \mathrm{~mol} \cdot \mathrm{m}^{6} \cdot \mathrm{s}^{-1} \cdot \mathrm{kg}^{-2}$; $\lambda_{0}=0.035 \mathrm{~W} \cdot \mathrm{m}^{-1} \cdot \mathrm{K}^{-1} ; \quad D_{0}=0.3 \mathrm{~cm}^{2} / \mathrm{s}, \quad$ это число взято таким, чтобы коэффициенты диффузии $D_{\mathrm{A}}=D_{\mathrm{B}}=0.3 \mathrm{~cm}^{2} / \mathrm{s}$ при температуре $T_{0}$ по порядку величины соответствовали экспериментальным данным [31,32].

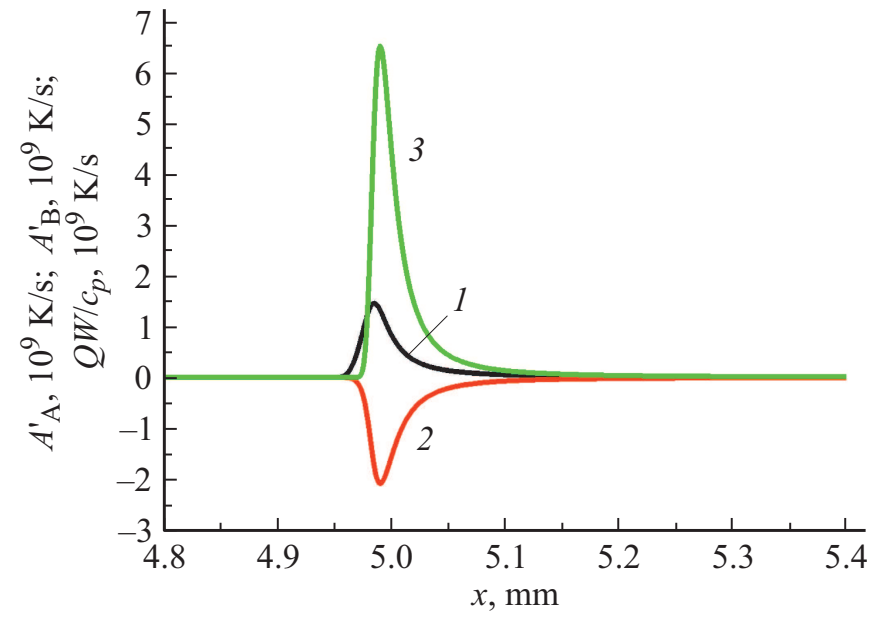

Рис. 1. Распределения мощностей источников тепла в зоне химической реакции: $A_{\mathrm{A}}^{\prime}$ (линия 1$), A_{\mathrm{B}}^{\prime}(2)$ и $Q W / c_{P}$ (3), для удобства они уменьшены в $10^{9}$ раз.

Численное решение уравнений из системы (10) проводилось применением неявной схемы [35], остальные детали расчетов приведены в [6]. Шаг интегрирования по пространственной координате $h=1 \mu \mathrm{m}$; шаг по времени $-\tau=2.5 \cdot 10^{-3} \mu \mathrm{s}$, их достаточно для фиксации сходимости численного метода. Ошибка аппроксимации производных по координате $x$ имеет порядок $h^{2}$.

Если в первом уравнении из (10) не учитывать производство химической работы $A_{\mathrm{A}}^{\prime}, A_{\mathrm{B}}^{\prime}$, то скорость пламени $u_{b}=11.6 \mathrm{~m} / \mathrm{s}$, температура пламени $T_{b}=3540 \mathrm{~K}$, $T_{b}=T(x \rightarrow \infty)$. Но в случае $A_{\mathrm{A}}^{\prime} \neq 0$ и $A_{\mathrm{B}}^{\prime} \neq 0$ наблюдается небольшое снижение скорости $u_{b}=11.15 \mathrm{~m} / \mathrm{s}$ и существенное снижение температуры $T_{b}=3317 \mathrm{~K}$, и большая разница $\Delta T_{b}=223 \mathrm{~K}$ не сильно отражается на скорости $u_{b}$. Ожидаемое уменьшение скорости пламени оценивается с помощью параметра $[22,27]$

$$
\frac{E \Delta T_{b}}{2 R T_{b}^{2}}
$$

Он равен 1.87 , тогда скорость $u_{b}$ должна была уменьшиться приблизительно в $\exp (1.87)=6.49$ раз. На самом деле она уменьшилась только в 1.04 раза. В упрощенной модели [6], где не учитывалось влияние состава газа на коэффициенты диффузии, включение химической работы приводило к обратному эффекту: $u_{b}$ и $T_{b}$ немного возрастали, и этот результат повторяется, если полагать $G_{\mathrm{A}}=G_{\mathrm{B}}=1$.

На рис. 1 показаны распределения $A_{\mathrm{A}}^{\prime}, A_{\mathrm{B}}^{\prime}$ и $Q W / c_{p}$ в зоне химической реакции, по отдельности $A_{\mathrm{A}}^{\prime}$ и $A_{\mathrm{B}}^{\prime}$ сравнимы по величине с $Q W / c_{p}$, но их знаки противоположны. Сумма $A_{\mathrm{A}}^{\prime}+A_{\mathrm{B}}^{\prime}<0$ и по абсолютной величине оказывается малой, поэтому химическая работа вносит относительно небольшой вклад в энергетический баланс реакции.

Профили температуры $T$ и концентрации $g_{\mathrm{A}}, g_{\mathrm{B}}$ имеют ярко выраженный передний фронт, за которым 


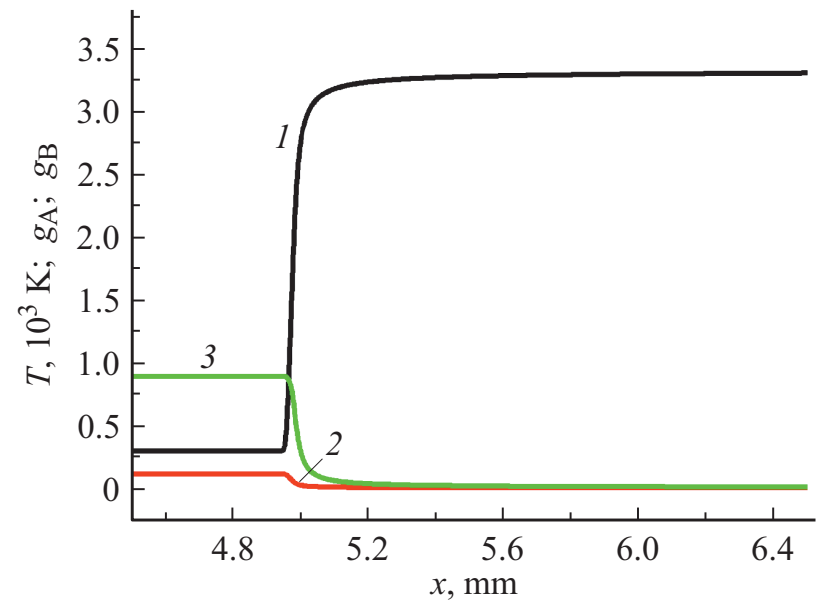

Рис. 2. Профили температуры и концентраций: $T$ (линия 1 ), $g_{\mathrm{A}}(2), g_{\mathrm{B}}(3)$.

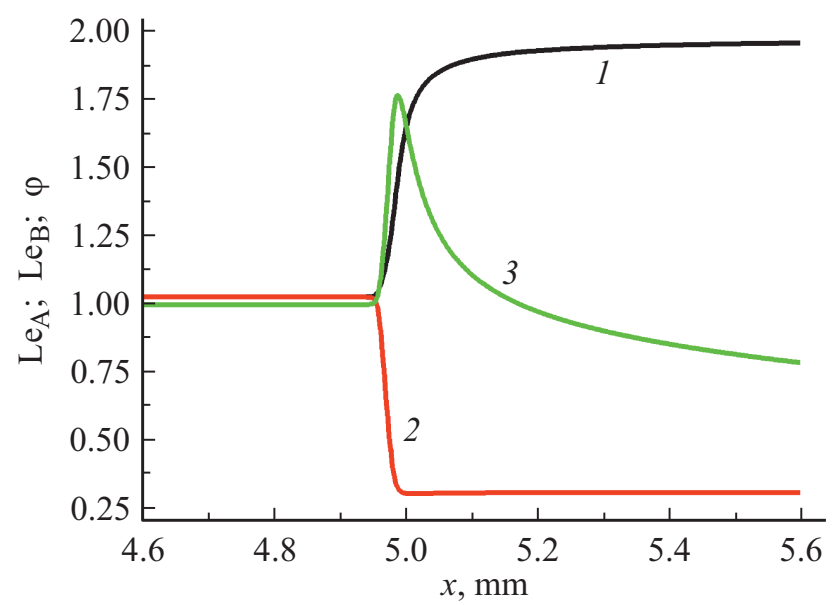

Рис. 3. Зависимости чисел Льюиса $\mathrm{Le}_{\mathrm{A}}$ (линия 1 ), $\mathrm{Le}_{\mathrm{B}}(2)$ и стехиометрического отношения $\varphi(3)$ от координаты $x$ в зоне химической реакции.

происходит плавный переход параметров к постоянным значениям в зоне пламени (рис. 2). Отношение чисел Льюиса (рис. 3):

$$
\mathrm{Le}_{\mathrm{A}}=\frac{\rho c_{p}}{\lambda} D_{\mathrm{A}}, \quad \mathrm{Le}_{\mathrm{B}}=\frac{\rho c_{p}}{\lambda} D_{\mathrm{B}}
$$

в свежей смеси составляет 1.03, но в зоне реакции возрастает до 6. Это сильное различие возникло из-за поведения функции $G_{\mathrm{A}}$ и $G_{\mathrm{B}}$ : до начала горения они равны, но в зоне пламени сильно различаются.

Их средние арифметические и средние геометрические значения примерно равны 1.18 и 0.65. Такие средние интересны тем, что они часто появляются в теории диффузии нескольких компонентов как их совместные характеристики, играющие важную роль для всего процесса $[16,17,19,22]$.

Влияние высокой скорости переноса молекул $\mathrm{H}_{2}(\mathrm{~A})$ на горение проявляется только в зоне химической реакции, впереди этой зоны большая подвижность молекул не имеет большого значения. Здесь они находятся в диффузионном равновесии с тяжелыми и малоподвижными молекулами $\mathrm{O}_{2}$ (В) и формируется их общий бинарный коэффициент $D_{\mathrm{A}}=D_{\mathrm{B}}$. В зоне химической реакции появляется третий компонент $\mathrm{H}_{2} \mathrm{O}(\mathrm{C})$, из-за этого диффузионное равновесие между реагентами $\mathrm{H}_{2}$ и $\mathrm{O}_{2}$ нарушается, поскольку молекулы $\mathrm{H}_{2}$ теперь переносятся в смеси $\mathrm{O}_{2} / \mathrm{H}_{2} \mathrm{O}$ с меньшей молярной массой, а молекулы $\mathrm{O}_{2}$ - в смеси $\mathrm{H}_{2} / \mathrm{H}_{2} \mathrm{O}$, имеющей теперь уже большую молярную массу.

Таким образом, водород оказывается в более легкой среде, а кислород - в более тяжелой. По этой причине на рис. 3 наблюдается рост $\mathrm{Le}_{\mathrm{A}}$ и снижение $\mathrm{Le}_{\mathrm{B}}$. Это обстоятельство приводит к сильному отклонению от 1 стехиометрического отношения (рис. 3)

$$
\varphi=\frac{v_{\mathrm{A}} \mu_{\mathrm{A}}}{v_{\mathrm{B}} \mu_{\mathrm{B}}} \frac{g_{\mathrm{B}}}{g_{\mathrm{A}}} .
$$

Результаты на этом рисунке показывают, что скорость химической реакции $W$ не достигает максимально возможного значения при $\varphi=1$ по этому параметру, реакция не протекает в идеальном режиме, но и не сильно от него отклоняется. Таким образом, повышение $\mathrm{Le}_{\mathrm{A}}$ и снижение $\mathrm{Le}_{\mathrm{B}}$ приводит к торможению реакции, но они не подавляют ее полностью. Этот транспортный механизм торможения реакции имеет чисто физическую природу, но не является единственно возможным в теории горения $[27,36]$.

\section{Заключение}

Предложенная в работе упрощенная теория для смеси из $N$ компонентов позволяет обходиться $N-1$ коэффициентами диффузии $D_{i}, i=1,2, \ldots$ К формулам для $D_{i}$ прилагаются способы их определения. В результате решение задач многокомпонентной диффузии сильно облегчается благодаря возможности применения закона Фика. В упрощенной теории перекрестные эффекты отсутствуют, они появляются только при переходе от объемных концентраций $r_{i}$ к массовым концентрациям $g_{i}$, но это уже не имеет принципиального значения, поскольку исследование горения можно проводить и с переменными $r_{i}$ вместо $g_{i}$.

Сравнение полученных здесь результатов моделирования горения смеси $\mathrm{H}_{2} / \mathrm{O}_{2}$ указывает на необходимость детального описания химического превращения. Это позволило бы получить более точную оценку вклада производства химической работы в энергетический баланс в зоне химической реакции. Появляющиеся при детальном моделировании различные производства химической работы частично уничтожают друг друга, в результате их суммарный эффект оказывается относительно небольшим по сравнению с тепловым эффектом химической реакции. Следующим важным фактором является учет зависимости коэффициентов диффузии от концентраций веществ. Транспортные явления в зоне 
химической реакции оказывают сильное влияние на производство химической работы и, как следствие, на температуру и скорость пламени.

\section{Конфликт интересов}

Авторы заявляют, что у них нет конфликта интересов.

\section{Список литературы}

[1] Г.Б. Манелис, Г.М. Назин, Ю.И. Рубцов, В.А. Струнин. Термическое разложение и горение взрывчатых веществ и порохов (Наука, М., 1996)

[2] Л.К. Гусаченко, В.Е. Зарко. ФГВ, 41 (1), 24 (2005). [L.K. Gusachenko, V.E. Zarko. Comb. Expl. Shock Waves, 41 (1), 20 (2005). DOI: 10.1007/s10573-005-0003-5]

[3] И.П. Базаров. Термодинамика (Лань, СПб., 2010)

[4] К.А. Путилов. Термодинамика (Наука, М., 1971)

[5] К.О. Сабденов. ФГВ, 57 (1), 51 (2021). [K.O. Sabdenov. Comb. Expl. Shock Waves, 57 (1), 46 (2021). DOI: $10.1134 / \mathrm{S} 0010508221010056]$

[6] К.О. Сабденов. ФГВ, 57 (2), 48 (2021). [K.O. Sabdenov. Comb. Expl. Shock Waves, 57 (2), 171 (2021). DOI: $10.1134 / \mathrm{S} 0010508221020052]$

[7] M.P. Burke, Yiguang Ju F.L. Dryer. 49th AIAA Aerospace Sciences Meeting, 4-7 Jan. 2011, Orlando, Florida. DOI: $10.2514 / 6.2011-93$

[8] A.L. Sanshez, F.A. Williams. Prog. in Energy Comb. Science, 41, 1 (2014).

[9] V. Giovangigli, L. Matuszewski, P. Gaillard. Progress in Astronautics and Aeronautics, 260, (2019), In press. ffhal02334430. https://hal.archives-ouvertes.fr/hal-02334430

[10] V.B. Betelin, V.F. Nikitin, E.V. Mikhalchenko. Acta Astronautica, 176, 628 (2020). DOI: 10.1016/j.actaastro.2020.03.051

[11] V.B. Betelin, B.V. Kryzhanovsky, N.N. Smirnov, V.F. Nikitin, I.M. Karandashev, M.Yu. Malsagov, E.V. Mikhalchenko. Acta Astronautica, 180, 58 (2021). DOI: $10.1016 /$ j.actaastro.2020.11.058

[12] Дж. Гиршфельдер, Ч. Кертисс, Р. Берд. Молекулярная теория газов и жидкостей (ИЛ, М., 1963) [J.O. Hirschfelder, Ch.F. Curtiss, R.B. Bird. Molecular Theory of Gases and Liquids (John Wiley and Sons, NY., 1954)]

[13] Дж. Ферцигер, Г. Капер. Математическая теория процессов переноса в газах (Мир, М., 1976) [J.H. Ferziger, H.G. Kaper. Mathematical Theory of Transport Processes in Gases (North-Holland Publishing Company, Amsterdam, London, 1972)]

[14] С.Ф. Василевский, И.А. Соколова, Г.А. Тирский. ПМТФ, 25 (4), 15 (1984). [S.A. Vasil'evskii, I.A. Sokolova, G.A. Tirskii. J. Appl. Mech. Tech. Phys., 25 (4), 510 (1984). DOI: $10.1007 / \mathrm{BF} 00909983$ ]

[15] И.А. Соколова. Математическое моделирование, 5 (5), 71 (1993).

[16] D. Matuszak, M.D. Donohue. Chem. Engin. Science, 60 (15), 4359 (2005).

[17] Dieter Bote. arXiv: 1007. 1775v1 [math. AP] 11 Jul 2010.

[18] S.H. Lam. Phys. Fluids, 18, 1 (2006). DOI: 10.1063/1.2221312

[19] R.B. Bird, D.J. Klingenberg. Advances in Water Resources, 62 (part B), 238 (2013).
[20] В.М. Жданов. ЖТФ, 89 (5), 646 (2019). [V.M. Zhdanov. Tech. Phys., 64 (5), 596 (2019).

DOI: $10.1134 / \mathrm{S} 106378421905027 \mathrm{X}]$

[21] В.М. Жданов, Г.А. Тирский. ПММ, 71 (5), 794 (2007). [V.M. Zhdanov, G.A. Tirskiy. J. Appl. Math. Mech., 71 (5), 718 (2007). DOI: 10.1016/j.jappmathmech.2007.11.005]

[22] Д.А. Франк-Каменецкий. Диффузия и теплопередача в химической кинетике (Наука, М., 1987) [D.A. FrankKamenetskii. Diffusion and Heat Transfer in Chemical Kinetics (Plenum Press, NY., 1969)]

[23] Т. Шервуд, Р. Пигфорд, Ч. Уилки. Массопередача (Химия, M., 1982) [T.K. Sherwood, R.L. Pigford, Ch.R. Wilke. Mass Transfer (McGraw-Hill, NY, 1975)]

[24] В.А. Кудинов. Техническая термодинамика (Высш. шк., M., 2000)

[25] О.Е. Александров, В.Д. Селезнев. ЖТФ, 86 (7), 26 (2016). [O.E. Aleksandrov, V.D. Seleznev. Tech. Phys., 61 (7), 982 (2016). DOI: 10.1134/S1063784216070033]

[26] Ф.А. Вильямс. Теория горения (Наука, М., 1971) [F.A. Williams. Combustion Theory (Westview Press, NY., 1985)]

[27] Я.Б. Зельдович, Г.И. Баренблатт, В.Б. Либрович, Г.М. Махвиладзе. Математическая теория горения и взрыва (Наука, M., 1980) [Ya.B. Zeldovich, G.I. Barenblatt, V.B. Librovich, G.M. Makhviladze. Mathematical Theory of Combustion and Explosion (Plenum, NY., 1985)]

[28] H.C. Lee, L.Y. Jiang, A.A. Mohamad. Int. J. Hydrogen Energy, 39, 1105 (2014).

[29] N. Kubota. Propellant and Explosives: Thermochemical Aspects of Combustion (Wiley VCH Verlag $\mathrm{GmbH} \& \mathrm{Co}$. KGaA, Veinheim, 2002)

[30] Б.В. Новожилов. Хим. физика, 7 (3), 388 (1988). [B.V. Novozhilov. Soviet J. Chem. Phys., 7 (3), 616 (1990).]

[31] К.О. Сабденов, М. Ерзада. ФГВ, 49 (6), 76 (2013). [K.O. Sabdenov, M. Erzada. Comb., Expl. Shock Waves, $49(6), 690$ (2013).]

[32] К.О. Сабденов, М. Ерзада. ФГВ, 52 (2), 76 (2016). [K.O. Sabdenov, M. Erzada. Comb., Expl. Shock Waves, $52(2), 193$ (2016).]

[33] Н.Б. Варгафтик. Справочник по теплофизическим свойствам жмидкостей и газов (Наука, М., 1972), изд. 2-е.

[34] Б.П. Никольский (ред.). Справочник химика (Химия, М.Л., 1982)

[35] Н.Н. Калиткин. Численные методы (Наука, М., 1978)

[36] Н.М. Эмануэль, Д.Г. Кнорре. Курс химической кинетики (Высшая школа, М., 1984) 